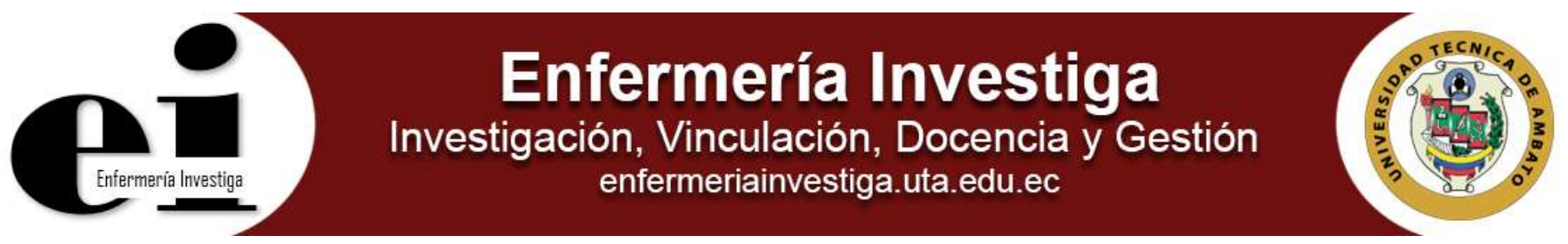

Artículo original

\title{
Atención prehospitalaria en emergencias por el Servicio Integrado de Seguridad ECU 911
}

Prehospital care in emergencies by the Integrated Security Service ECU 911

Paul Sebastián Alulema Zurita1, Ronelsys Martínez Martínez², Raúl González Salas², Gustavo Moreno Martín², Jacqueline Cisneros Pérez ${ }^{4}$

\author{
${ }^{1}$ Fiscalía General del Estado, Santo Domingo de los Tsáchilas, Ecuador. \\ ${ }^{2}$ Carrera de Medicina, Facultad de Ciencias Médicas, Universidad Regional Autónoma de los Andes, Ambato, Ecuador. \\ ${ }^{3}$ Carrera de Enfermería, Facultad Ciencias de la Salud, Universidad Técnica de Ambato, Carrera de Medicina Facultad de Ciencias Médicas, Universidad Regional \\ Autónoma de los Andes, Ambato, Ecuador. Investigador Auxiliar.
}

${ }^{4}$ Carrera de Estimulación Temprana, Facultad Ciencias de la Salud, Universidad Técnica de Ambato, Ambato, Ecuador.

Alulema ZPS, Martínez MR, González SR, Moreno MG, Cisneros PJ. Atención prehospitalaria en emergencias por el Servicio Integrado de Seguridad ECU 911. Enferm Inv (Ambato). 2017; 2(2):50-53.

2477-9172 / 2550-6692 Derechos Reservados @ 2017 Universidad Técnica de Ambato, Carrera de Enfermería. Este es un artículo de acceso abierto distribuido bajo los términos de la Licencia Creative Commons, que permite uso ilimitado, distribución y reproducción en cualquier medio, siempre que la obra original es debidamente citada.

\section{Historia:}

Recibido: 08 mayo 2017

Revisado: 09 mayo 2017

Aceptado: 10 mayo 2017

Palabras Claves: Atención prehospitalaria; emergencia; Servicio Integrado de

Seguridad ECU 911; tiempo de respuesta

Keywords: Prehospital care; emergency, Integrated Security Service ECU 911; response time

\section{Resumen}

Introducción: La atención médica que se brinda previa al ámbito hospitalario, implica la coordinación de una serie de acciones y toma de decisiones por parte del personal que la ejecuta. El Servicio Integrado de Seguridad ECU 911, coordina la atención prehospitalaria en el territorio nacional, se ha ido ampliando con el transcurso del tiempo y ha mejorado la cobertura sobre el territorio nacional. Objetivo: Determinar la situación actual de la atención de emergencias prehospitalaria en relación con el tiempo de respuesta y a los eventos reportados al Servicio Integrado de Seguridad ECU 911 de la ciudad de Ambato.

Métodos: Se realizó un estudio observacional descriptivo y retrospectivo con universo de 145 profesionales que laboran en el servicio y las 1247 atenciones registradas de enero a junio de 2015 , no se seleccionó muestra.

Resultados: Se encontró que el tiempo de respuesta del sistema es susceptible de ser mejorado, sobre la base de un personal mayormente joven, que se encuentra motivado por la labor que realiza, pero que sin embargo desconoce en gran medida los protocolos establecidos para la función que realizan, conllevando a poca adherencia a estos.

Conclusiones: La atención prehospitalaria fue mayoritariamente a urgencias o emergencias de tipo quirúrgicas o traumatológicas. El tiempo de respuesta del sistema fue susceptible de ser mejorado acorde a estándares internacionales. La mayoría del personal presentó poco tiempo laborando dicha área debiendo mejorarse la adherencia a protocolos de atención prehospitalaria.
\end{abstract}

\begin{abstract}
Introduction: The medical care provided prior to the hospital environment, involves the coordination of a series of actions and decision making by the staff that executes it. The Integrated Security Service ECU 911 coordinates prehospital care in the national territory, has been expanded over time and improved coverage over the national territory.

Objetive: To determine the current situation of the prehospital emergency care in relation to the response time and to the events reported to the Integrated Security Service ECU 911 of the city of Ambato.

Methods: A descriptive and retrospective observational study was carried out with a universe of 145 professionals working in the service and 1247 appointments recorded from january to june 2015, no sample was selected.

Results: It was found that the response time of the system is susceptible to improvement, based on a mostly young staff, who is motivated by the work that he does, but which, however, is largely unaware of the protocols established for the function performed, leading to low adherence to these.

Conclusions: The prehospital care was mostly to emergencies or emergencies of the surgical or trauma type. The response time of the system was able to be improved according to international standards. The majority of the staff presented a short time working in this area and the adherence to prehospital care protocols should be improved.
\end{abstract}




\section{Introducción}

La atención en urgencias y emergencias tiene la particularidad de comenzar en el sitio donde ocurren los hechos, o sea en la comunidad, una vez sucede el evento que amenaza la salud de los individuos. Esta atención médica que se brinda previa al ámbito hospitalario, implica la coordinación de una serie de acciones y toma de decisiones por parte del personal que la ejecuta, lo cual define su nivel de complejidad ${ }^{1}$.

Las experiencias clásicas griegas y romanas con sus primitivos servicios de carros ambulancia constituyen las primeras imágenes históricas fuertes sobre la actividad de la emergencia médica.

En la edad moderna son los novedosos servicios de recogida y clasificación de heridos del ejército napoleónico los que marcan el inicio de un largo recorrido en el que los hitos más importantes desde el punto de vista logístico y asistencial $^{2}$.

El avance más significativo que impulso los servicios de emergencias extrahospitalarias fue la descripción por primera vez del masaje cardiaco en 1960 por Kouwenhoven y desde entonces las maniobras de Reanimación CardioPulmonar Cerebral (RCPC) se han generalizado ${ }^{3}$.

La primera ambulancia con un desfibrilador portable, fue puesta en marcha en 1965 por Frank Pantridge en el Royal Victoria Infirmary en Belfast, creando así la primera unidad de emergencia extrahospitalaria de cuidados coronarios. Este concepto fue rápidamente adoptado en Estados Unidos y el resto del mundo.

Pero evidentemente, son los servicios civiles, inicialmente en EEUU y posteriormente en Europa, en los que se manifiesta el profundo avance en la implantación de todo tipo de unidades asistenciales para la emergencia médica.

En 1960 la Academia Nacional de Ciencias introdujo normas para el entrenamiento del personal que tripula las ambulancias y en 1962 se programó el primer curso para la formación de Técnicos en Emergencias Médicas. Los primeros esfuerzos desarrollados estuvieron encaminados a las enfermedades coronarias como las arritmias graves y muerte súbita, fue así como aparecieron la primeras Unidades Coronarias Móviles ${ }^{4}$.

A mediados de los años 50 del siglo pasado comenzaban a surgir dos modelos de Sistemas de Emergencias Médicas, ambos enmarcados en la atención médica prehospitalaria. Así comienza el destaque del modelo norteamericano, el mismo priorizaba la atención en el lugar de los hechos, brindada por personal paramédico preferentemente; de otro lado el modelo francés, este valoraba el traslado del individuo al ámbito hospitalario para su manejo, preferentemente personal médico sería el encargado de brindar dicha atención ${ }^{5}$.

Las características entes mencionadas son las más distintivas de ambos modelos de Sistemas de Emergencia Médicas, sin embargo ambos presentaron significativas limitaciones. En la actualidad se considera necesario tomar los elementos positivos de ambos sistemas, lo que implica una adecuada toma de decisiones en el lugar de los hechos hasta el individuo sea trasladado al escenario hospitalario.

En el siglo XIX empieza a escribirse la historia de la atención prehospitalaria latinoamericana, es así que en el año 1979 el Dr. Gustavo Báez Cirujano de la Cruz Roja Mexicana intenta comenzar un Programa de Entrenamiento sin éxito ${ }^{6}$.
El mismo año en Brasil y Venezuela inician sus pasos en la creación de los mismos programas. En 1981 los doctores Griffe y Zamudio retoman el tema y crean la Escuela Formal de Técnicos en Urgencias Médicas en México. El mismo año en República Dominica que ya contaba con sus avances crea el Primer Curso Emergency Medical Technician (EMT) $)^{7}$.

Desde la aparición de la Cruz Roja Ecuatoriana en Abril de 1910 se inició la atención prehospitalaria de manera rudimentaria sin personal capacitado ya que la mayoría de sus integrantes se unieron de forma voluntaria ${ }^{8}$. Con el transcurso del tiempo esta entidad se encargó de capacitar a sus voluntarios y crear el Instituto de la Cruz Roja, el mismo que se encarga de la preparación de los nuevos Técnicos en Emergencias y Desastres (Paramédico), esta entidad en conjunto con Cuerpo de Bomberos cubrían determinados eventos emergentes, pero con el aumento de la población se vieron limitados sus trabajos y esfuerzos por atender a todos los eventos a nivel nacional, es así como surge la necesidad por parte del gobierno nacional en el año 2012, el Sistema Nacional de Salud establece a la Atención Prehospitalaria como un nivel autónomo e independiente, donde el personal de la misma (medico, paramédico o enfermera) ejerce una labor primordial para el correcto funcionamiento del mismo, en cada uno de los niveles de atención prehospitalaria ${ }^{9}$.

En el Ecuador, la atención prehospitalaria ha tenido un desarrollo limitado, ha estado orientado más al sector privado que al sector público, y hasta hace 3 años se limitaba únicamente a las entidades de Cruz Roja y Cuerpo de Bomberos quienes cubrían todo tipo de emergencias prehospitalarias. En la actualidad el Servicio Integrado de Seguridad ECU 911, es el encargado de la coordinación de la atención prehospitalaria en el territorio nacional, servicio que se ha ido ampliando con el transcurso del tiempo y a su vez mejorando la cobertura sobre el territorio nacional. En este sistema gracias a la llamada única con la línea 911 en la cual se reporta todo tipo de emergencias las mismas que se encaminan para las diferentes instituciones responsables de las mismas; cabe recalcar que las instituciones de primera respuesta en atención prehospitalaria son Ministerio de Salud Pública, Cuerpo de Bomberos y Cruz Roja9 ${ }^{9}$.

Estas instituciones cuentan con vehículos de primera respuesta entre los cuales están ambulancias equipadas de acuerdo a los diferentes niveles de complejidad. Dichas ambulancias, según la normativa legal vigente, deben integrar en su personal por lo menos un profesional de salud (medico, paramédico o enfermera) quien estará a cargo de la emergencia y cumplirá con conocimientos básicos de atención pre-hospitalaria y manejo de emergencias y desastres ${ }^{10}$.

El objetivo del presente artículo fue determinar la situación actual de la atención de emergencias prehospitalaria en relación con el tiempo de respuesta y a los eventos reportados al Servicio Integrado de Seguridad ECU 911 de la ciudad de Ambato.

\section{Materiales y Métodos}

El estudio fue desarrollado en el cantón Ambato, capital de la provincia Tungurahua. Ubicada sobre unos 2500 metros sobre el nivel del mar, Ambato cuenta con una población superior a los 300 mil habitantes. El Centro Zonal de Seguridad Ambato se encuentra en la Av. Albert Einstein, Km 1 Vía a Techo Propio, sector Pishilata.

Se desarrolló un estudio, observacional, descriptivo y retrospectivo con el objetivo de diagnosticar la atención 
prehospitalaria que brinda el Servicio Integrado de Seguridad ECU 911 de la ciudad de Ambato. El universo de estudio estuvo conformado por los 145 profesionales que laboran en el servicio y las 1247 atenciones registradas en el sistema de enero a junio de 2015. No se seleccionó muestra.

\section{Recolección y procesamiento de datos.}

Fueron utilizados los datos provenientes de los registros de servicio del Servicio Integrado de Seguridad ECU 911. Se aplicó una encuesta a los profesionales con la finalidad de obtener información referente al tiempo laborando en el sistema, condiciones de trabajo y conocimiento de los protocolos establecidos para la atención prehospitalaria. Se confeccionó una base de datos en Microsoft SPSS-22.0. Se utilizaron métodos de estadística descriptiva para el trabajo de las variables.

\section{Resultados}

La respuesta del sistema tardó entre 11 y 20 minutos en casi la mitad de las veces que fue activado ( $n=61249.1 \%$ ). El promedio del tiempo de respuesta fue de 18 minutos (tabla 1).

Tabla 1. Tiempo de respuesta del Servicio Integrado de Seguridad ECU 911. Ambato. Enero-junio de 2015.

\begin{tabular}{lcc}
\hline Tiempo de respuesta (minutos) & $\mathbf{n}$ & $\%$ \\
\hline$\leq 10$ & 90 & 7.2 \\
$\geq 11$ y $\leq 20$ & 612 & 49.1 \\
$>20$ & 545 & 43.7 \\
\hline Total & $\mathbf{1 2 4 7}$ & $\mathbf{1 0 0}$ \\
\hline
\end{tabular}

Fuente: Registros de atención Servicio Integrado de Seguridad ECU 911.

Los traumatismos, accidentes de tránsito y emergencias quirúrgicas fueron los principales motivos de activación del sistema en el periodo de estudio. Las urgencias/emergencias clínicas o médicas se ubicaron en segundo lugar. Un pequeño número de asistencias correspondieron con medicina ambulatoria, en muchos casos tratándose de urgencias sentidas y no verdaderas urgencias (tabla 2).

Tabla 2. Distribución de pacientes atendidos por el Servicio Integrado de Seguridad ECU 911 según especialidad médica. Ambato. Enero-junio de 2015.

\begin{tabular}{lcc}
\hline Especialidad Médica & $\mathbf{n}$ & $\mathbf{\%}$ \\
\hline Cirugía-Traumatología & 380 & 30.5 \\
Medicina Interna & 297 & 23.8 \\
Ginecología & 126 & 10.1 \\
Pediatría & 109 & 8.7 \\
Medicina Ambulatoria & 101 & 8.1 \\
Urología & 91 & 7.3 \\
Otras & 143 & 11.5 \\
\hline Total & $\mathbf{1 2 4 7}$ & $\mathbf{1 0 0}$ \\
\hline
\end{tabular}

Se encontró un personal joven a cargo de la atención prehospitalaria en su mayoría, fundamentalmente por ser el Sistema Integrado de Seguridad ECU 911 un sistema relativamente joven desde su creación (tabla 3).

En la tabla 4 se muestra el tiempo de labor vinculado a atención prehospitalaria por parte de los profesionales a cargo del Servicio Integrado de Seguridad ECU 911, la mayoría ha estado por espacio menor a cinco años.
Tabla 3. Distribución según edad del personal que labora en el Servicio Integrado de Seguridad ECU 911. Ambato. Enero-junio de 2015.

\begin{tabular}{lcc}
\hline Edad (años) & $\mathbf{n}$ & \% \\
\hline$\geq 20$ y $\leq 30$ & 43 & 29.7 \\
$\geq 31$ y $\leq 40$ & 63 & 43.4 \\
$\geq 41$ y $\leq 50$ & 27 & 18.6 \\
$>50$ & 12 & 8.3 \\
\hline Total & $\mathbf{1 4 5}$ & $\mathbf{1 0 0}$ \\
\hline
\end{tabular}

Tabla 4. Tiempo que labora en el ECU 911 u otro Sistema de Atención Prehospitalaria. Ambato. Enero-junio de 2015.

\begin{tabular}{lcc}
\hline Tiempo laborando (años) & $\mathbf{n}$ & $\%$ \\
\hline$\leq 2$ & 71 & 48.9 \\
$\geq 3$ y $\leq 5$ & 62 & 42.8 \\
$>5$ & 12 & 8.3 \\
\hline Total & $\mathbf{1 4 5}$ & $\mathbf{1 0 0}$ \\
\hline Fuente: Encuesta a profesionales del Servicio.
\end{tabular}

En lo referente a las condiciones de trabajo se pudo constatar que son calificadas de buenas por la mayoría del personal que la labora en el Servicio Integrado de Seguridad ECU 911 (tabla 5).

Tabla 5. Valoración de las condiciones de trabajo. Servicio Integrado de Seguridad ECU 911. Ambato. Enero-junio de 2015.

\begin{tabular}{lcc}
\hline Condiciones de trabajo & $\mathbf{n}$ & $\%$ \\
\hline Buenas & 92 & 63.4 \\
Regulares & 41 & 28.3 \\
Malas & 12 & 8.3 \\
\hline Total & $\mathbf{1 4 5}$ & $\mathbf{1 0 0}$ \\
\hline Fuente: Encuesta a profesionales del Servicio. &
\end{tabular}

\section{Discusión}

Tratándose de un cantón que no se caracteriza por una gran extensión superficial y con zonas de difícil acceso, se puede afirmar que el tiempo de respuesta es susceptible de ser mejorado. En un estudio reciente desarrollado en la Cuidad de Medellín, Colombia, se estimó que el tiempo de respuesta promedio fue de 14 minutos, y el $30 \%$ de las respuestas se obtuvieron en un tiempo de hasta 10 minutos; esto contrasta con la presente investigación, donde a pesar de tratarse de una ciudad mucho más pequeña, solo el $7,2 \%$ de respuestas ocurrieron en los 10 minutos que siguen a la activación del sistema ${ }^{11}$.

En la bibliografía revisada se encontró que en Latinoamérica los accidentes de tránsito y traumatismos constituyen la causa fundamental que motiva la activación de sistemas médicos de atención prehospitalaria ${ }^{12,13}$. Sin embargo esto contrasta con países desarrollados con sólidos sistemas de salud donde las urgencias o emergencias clínicas son la primera causa. Tal es el caso del estudio "Rendimiento e impacto de los primeros respondedores en la evolución de la medicina de emergencias prehospitalaria en Suiza"; en este caso las urgencias/emergencias de tipo quirúrgicas acapararon el primer lugar, desplazando a las clínicas, y las correspondientes con medicina ambulatoria ocuparon un tercer lugar. Cabe señalar que en estas latitudes, con sistemas médicos de emergencias bien establecidos, la población cada vez hace más uso de ellos por su alto poder resolutivo, lo que motiva muchas atenciones sin que realmente se trate de una urgencia o emergencia ${ }^{14}$.

A nivel mundial la población que labora en estos sistemas es joven, por las características de la atención que se brinda $y$ por el auge que ha tenido la medicina de urgencias y 
emergencias en los últimos años ${ }^{15}$, el presente estudio confirmó esa tendencia.

Al tratarse de un sistema relativamente joven, más de la mitad del personal que labora en él ha tenido una trayectoria menor a dos años en la atención médica prehospitalaria. Solo un 8,3\% rebasó los 5 años, se trató en estos casos de profesionales que se encontraron vinculados a estos servicios con antigüedad en hospitales, y otros que al iniciarse el ECU 911, por tener perfil a fin se incorporaron desde su apertura. Otros autores han reportado resultados similares en cuanto al personal que labora en estos sistemas médicos de atención prehospitalaria ${ }^{16}$.

El $28 \%$ de los profesionales encuestados en el Sistema Integrado de Seguridad ECU 911, refirieron que en ocasiones las condiciones de ambulancias no son las mejores, algo que es susceptible de ser mejorado, sobre todo teniendo en cuanta que el presupuesto asignado es suficiente para ofrecer un servicio de calidad. Un estudio similar realizado en el Ecuador en el año 2014 reportó como se ha ido ganando espacio desde el punto de vista organizacional, por la complejidad que presume un servicio de salud de este tipo, además a como se ha ganado en cuanto a las condiciones laborales y la atención al hombre ${ }^{17}$.

Resaltó el hecho que la mayoría de los profesionales $(n=126$ $87,9 \%$ ) refirió no conocer los protocolos establecidos para brindar la atención médica prehospitalaria en el Sistema Integrado de Seguridad ECU 911. Esta realidad debe ser transformada toda vez que la atención de urgencias o emergencias se desarrolla con tiempo limitado, muchas veces en el curso de compromiso vital del paciente, debiendo tomarse decisiones oportunas en aras de optimizar el tiempo y el manejo del paciente; todo lo cual da origen a una atención protocolizada y contextualizada en dependencia de la ubicación del sistema de emergencias ${ }^{18}$.

\section{Conclusiones}

EI Servicio Integrado de Seguridad ECU 911 brinda una atención prehospitalaria en el cantón Ambato, mayoritariamente a urgencias o emergencias de tipo quirúrgicas-traumatológicas. El tiempo de respuesta del sistema fue susceptible de ser mejorado acorde a estándares internacionales. Al tratarse de un sistema de atención prehospitalaria relativamente joven, la mayoría del personal presentó poco tiempo laborando dicha área. La adherencia a protocolos de atención prehospitalaria fue baja, situación que no es compatible con la atención a urgencias o emergencias.

\section{Referencias}

1. Avery P, Salm L, Bird F, Hutchinson A, Matthies A, Hudson A, et al. London Trauma Conference 2015: London, UK, 8-11 December 2015. Scand J Trauma Resusc Emerg Med [en línea]. 2016;24(Suppl 1):78. Disponible

http://www.ncbi.nlm.nih.gov/pmc/articles/PMC4928155/

2. Ugarte Taboada C. Historia de los Servicios de Emergencia de Lima y Callao. Rev Medica Hered [en línea]. 2000 [citado 19 de mayo de 2017];11(3):97-106. Disponible http://www.scielo.org.pe/scielo.php?pid=S1018 $130 \times 2000000300005 \&$ script $=$ sci arttext

3. Rodríguez AP, García AÁ, Gāeo FH, Moraza AS Servicios de emergencia médica extrahospitalaria en España (II). Emergencias [en línea]. 1998 [citado 19 de mayo de 2017];10(4):245. Disponible en: http://www.medynet.com/usuarios/jraguilar/historia.pdf

4. Holtermann KA, González AGR. Desarrollo de sistemas de servicios de emergencias médicas: experiencia de los Estados Unidos de América para países en desarrollo, octubre 2003, Washington [en línea]. Pan American Health Org; 2003. 252 p. Disponible en:
https://books.google.es/books?hl=es\&lr=\&id=5Bi1i95T\&kUC\&oi=fnd\& $\mathrm{pg}=\mathrm{PA} 1 \& \mathrm{dq}=$ Desarrollo+de+sistemas+de+servicios+de+emergencia s+m\% C3\%A9dicas:+experiencia+de+los+Estados+Unidos+de+Am\% C3\%A9rica+para+pa\%C3\%ADses+en+desarrollo,+octubre+2003,+W ashington\&ots=njCwoHE0qf\&sig=P6wOrU3A1eklyEIsCliCXE7MWzo \#v=onepage\&q=Desarrollo\%20de\%20sistemas\%20de\%20servicios $\% 20 \mathrm{de} \% 20$ emergencias $\% 20 \mathrm{~m} \% \mathrm{C} 3 \%$ A9dicas $\% 3 \mathrm{~A} \% 2$ experiencia $\%$ 20de $\% 20$ los $\% 20$ Estados $\% 20$ Unidos $\% 20$ de $\% 20$ Am $\%$ C3\%A9rica $\% 20$ para $\% 20$ pa $\%$ C3\%ADses $\% 20$ en $\% 20$ desarrollo\%2C\%20octubre $\% 202$ $003 \% 2 \mathrm{C} \% 20$ Washington\&f=false

5. Véliz Martínez PL, Jorna Calixto AR. Evolución histórica y perspectivas futuras de la Medicina Intensiva y Emergencia como especialidad en Cuba. Educ Médica Super [en línea]. 2014 [citado 19 de mayo de 2017];28(3):592-602. Disponible en: http://scielo.sld.cu/scielo.php?pid=S0864-

$21412014000300018 \&$ script=sci arttext\&tIng=en

6. Garnica González TM, Mena González LM, Moreno Ríos JJ. Estudio comparativo de los modelos de atención prehospitalaria entre Colombia y México [en línea]. 2017 [citado 19 de mayo de 2017]. Disponible

http://bdigital.ces.edu.co:8080/repositorio/handle/10946/4481

7. Garlet ER, da Silva Lima MAD, dos Santos JLG, Marques GQ. Finalidade do trabalho em urgências e emergências. Rev Lat Am Enfermagem [en línea]. 2009 [citado 19 de mayo de 2017];17(4):535540. Disponible en: http://www revistas usp . br/rlae/article/view/4037

8. Panamericana OS. Sociedades e instituciones científicas de la América Latina. 1939 [citado 19 de mayo de 2017]; Disponible en: http://iris.paho.org/xmlui/bitstream/handle/123456789/1092/MIMEOP B084.pdf?sequence=1

9. Zea S, Miroslava, Lady. Tipología para homologar establecimientos de salud por niveles. 2016 [citado 19 de mayo de 2017]; Disponible en: http://repositorio.uta.edu.ec/handle/123456789/21725

10. Caballería I. Descripción de los niveles de atención sanitaria. Atención primaria: Centro de Salud, organización, funciones, control de calidad. Atención especializada: Organización funciones, control de calidad. Ssitemas de información y registro. [en línea]. 2013 [citado 19 de mayo de 2017]. Disponible en: https://riunet.upv.es/handle/10251/29953

11. Cañaveral MNE. Tiempo de respuesta del servicio de APH del cuerpo de bomberos en la ciudad de Medellín año 2010 y su asociación con la satisfacción del usuario. Unaciencia [en línea]. 1 de julio de 2015 [citado 19 de mayo de 2017];0(6). Disponible en: http://revistas.unac.edu.co/index.php/Unaciencia/article/view/97

12. Haagsma JA, Graetz N, Bolliger I, Naghavi M, Higashi H, Mullany EC, et al. The global burden of injury: incidence, mortality, disabilityadjusted life years and time trends from the Global Burden of Disease study 2013. Inj Prev [en línea]. febrero de 2016;22(1):3-18. Disponible en: http://www.ncbi.nlm.nih.gov/pmc/articles/PMC4752630/

13. Ordoñez CA, Morales M, Rojas-Mirquez JC, Bonilla-Escobar FJ, Badiel M, Miñán Arana F, et al. Trauma Registry of the Pan-American Trauma Society: One year of experience in two hospitals in southwest Colombia. Colomb Médica CM [en línea]. 1 de septiembre de 2016;47(3):148-54. Disponible en: http://www.ncbi.nlm.nih.gov/pmc/articles/PMC5091273/

14. Urwyler N, Theiler L, Schönhofer J, Kämpfen B, Stave C, Greif R. Rendimiento e impacto de los primeros respondedores en la evolución de la medicina de emergencias prehospitalaria en Suiza. Emergencias [en línea]. 2012 [citado 19 de mayo de 2017];24:426-32. Disponible en:

https://www.researchgate.net/profile/Lorenz_Theiler/publication/2772 61461 Rendimiento e impacto de los primeros respondedores e n_la_evolucion de $\bar{l}$ - medicina de emergencias prehospitalaria_e n_Suiza/links/55e430e308aecb1a7cc9f1c3.pdf

15. Padilla Tejeda CW. Evaluación del servicio de atención prehospitalaria en la ciudad de Cartagena frente el manejo del paciente con politraumatismo atendidos por el Centro Regulador de Urgencias y Emergencias (CRUE) durante el año 2014. 2014; Disponible en: http://siacurn.app.curnvirtual.edu.co:8080/xmlui/handle/123456789/8 44

16. Cano-del Pozo MI, Obón-Azuara B, Valderrama-Rodríguez M, RevillaLópez C, Brosed-Yuste C, Fajardo-Trasobares E, et al. Las emergencias extrahospitalarias ante la cultura de seguridad. Rev Calid Asist [en línea]. septiembre de 2014 [citado 19 de mayo de 2017];29(5):263-9. Disponible http//linkinghub.elsevier.com/retrieve/pii/S1134282X14000761

17. Santos PSB, Sierra JPC. Los servicios de emergencias médicas en el Ecuador: una tarea pendiente. Rev Fac Cienc Médicas [en línea]. 2016 [citado 19 de mayo de 2017];32(3):58-69. Disponible en: http://192.188.48.56/ojs/index.php/medicina/article/view/897

18. Julián A. Manual de Protocolos y Actuación en URGENCIAS de Complejo Hospitalario de Toledo [en línea]. Complejo Hospitalario de Toledo; 2014. Disponible en: https://dialnet.unirioja.es/servlet/libro?codigo $=563012$ 